\title{
Terrestrial planet formation in exoplanetary systems with a giant planet on an external orbit
}

\author{
P. Thébault ${ }^{1}$, F. Marzari ${ }^{2}$, and H. Scholl ${ }^{3}$ \\ 1 Observatoire de Paris, Section de Meudon, 92195 Meudon Principal Cedex, France \\ 2 Dipartimento di Fisica, Universita di Padova, Via Marzolo 8, 35131 Padova, Italy \\ 3 Observatoire de la Cote d'Azur, Dept. Cassini, URA CNRS 1362, BP 229, 06304 Nice, France
}

Received 10 July 2001 / Accepted 28 November 2001

\begin{abstract}
The Epsilon Eridani and 47 UMa extrasolar systems both have moderately massive planets orbiting relatively far away from the central star (at 3.3 and beyond $2.1 \mathrm{AU}$, respectively). This peculiarity makes them possible candidates for harboring terrestrial planets in their inner regions since the external giant planet might not have inhibited planet growth in the neighborhood of the star. Here we numerically investigate how the accretion of terrestrial planets may have been affected by the presence of the detected giant planets in external orbits. The uncertain timing of the giant planet formation with respect to the planetesimal accumulation process lead us to consider two possible scenarios: 1) the perturbing massive planet was fully formed when the planetesimals in the inner zone were still in their early phases of accumulation, and 2) the giant planet reached its final mass only later on when lunar-sized embryos were already present in the inner disk. In the first case we have used a deterministic numerical algorithm to compute the relative velocity distribution in a swarm of planetesimals affected by the gravitational perturbations of the giant planet and, possibly, by gas drag. The second scenario is explored by using the symplectic algorithm by Chambers (1999) to simulate the late stage of terrestrial planet formation. The Epsilon Eridani system turns out to be a very hostile environment for planetary formation in the terrestrial region when the giant planet formed early. The planet's high eccentricity (0.608) induces very large relative velocities between planetesimals in inner orbits and collisions result mostly in disruption rather than accretion. On the other hand, if large embryos had enough time to form before the completion of the giant planet, they can accrete each other in giant impacts and coalesce into a few terrestrial planets within 0.8 AU from the star. For the 47 UMa system the situation is quite similar. The large mass of the inner giant planet excites large eccentricities among the planetesimals in the terrestrial region preventing accumulation. However, the damping effect on the relative velocities caused by gas drag opens a chance for accretion in a small region around the star inside 0.3 AU. The second and more distant planet recently discovered around 47 Uma does not significantly affect the encounter velocity distribution in the inner regions of the system. As for Epsilon Eridani, orderly growth of lunar-sized embryos takes place within $0.8 \mathrm{AU}$ in presence of a late-formed giant planet. In either case, beyond 0.8 AU planetesimals or planetary embryos are rapidly removed by the massive planet perturbations.
\end{abstract}

Key words. stars: planetary systems - planetary systems: formation - planets and satellites: formation

\section{Introduction}

Most of the over 60 extrasolar planets discovered up to date are Jovian-type planets and are on orbits relatively close to the central star. It is believed that these planets formed at larger distances possibly in the conventional way by gas accretion onto a solid icy/rocky core (Pollack et al. 1996) and then migrated inwards either by planetesimal scattering (Murray et al. 1998), or by tidal interaction with the evolving circumstellar disk (Ward \& Hourigan 1989; Lin et al. 1996), or by gravitational scattering with other planets (Weidenschilling \& Marzari 1996). Conceivably, these massive planets depleted during

Send offprint requests to: $\mathrm{P}$. Thébault, e-mail: philippe.thebault@obspm.fr their migration the region of the disk outside their final orbit, and eventually prevented the formation of terrestrial planets in the habitable zone inside their final orbit. It is possible that the subsequent evolution of the circumstellar disk supplied the habitable zone with additional material for planet formation. Even in this case the strong gravitational perturbations by the inner planet might have halted the process of planetesimal accretion by favoring destructive collisions between the planetesimals.

Recently, a few exoplanets have been found farther away from the star. 47 Uma, 14 Her, HD 190228 , HD 10697, HD 50554, HD 106252, and Epsilon Eridani are all systems with a single massive planet orbiting beyond 2 AU. They resemble our solar system more closely and it is natural to ask whether these systems can harbor 
terrestrial planets in their inner regions. At present, we cannot give a definitive answer to this question since the detection of terrestrial planets is beyond the capabilities of our telescopes. However, future space missions like COROT (ESA), DARWIN (ESA) or Terrestrial Planet Finder (NASA) will be able to explore remote star systems to find signatures of terrestrial planets. In the target selection phase of these missions should we give priority to those star systems where a giant planet has already been detected, or should we search elsewhere to maximize the detection efficiency? To address this problem we have to investigate theoretically whether terrestrial planets can form against the gravitational perturbations of a giant gaseous planet on putative planetesimals populating the terrestrial region.

Jones et al. (2001) have recently analyzed the problem of orbital stability in the habitable regions of some of these extrasolar systems. In particular they have shown that the 47 Uma system could harbor a terrestrial planet in an orbit that remains confined to the habitable zone. However, these simulations focus on the orbital stability problem, and do not investigate the possibility that such terrestrial planets can actually form in these systems.

The process of planetesimal accumulation into planets depends on the complex interactions between the mutual gravitational perturbations among the planetesimals, mutual collisions, and the possible presence of gas drag. One of the crucial parameters for an efficient growth of planetesimals is the average value of the relative velocity $\Delta v$ between two colliding bodies. In our own solar system, it is believed that the $\Delta v$ was low, i.e. of the order of the escape velocities of the dominant kilometer-sized planetesimal population, for a large fraction of the accretion history. Such low velocities favored the rapid runaway growth of isolated planetary embryos on timescales of the order of $10^{5}$ yrs (e.g. Lissauer 1993). However, it is known that the gravitational perturbations of a giant planet act to increase relative velocities among planetesimals so that erosion can prevail instead of growth when two bodies collide. This kind of problem has been investigated for the Solar System by Thébault \& Brahic (1999), Kortenkamp \& Wetherill (2000) and Charnoz et al. (2001). These studies showed that early-formed Jupiter and Saturn can perturb the planetesimal accumulation process in the Earth region and, eventually, prevent the formation of a terrestrial planet in the asteroid belt. In extrasolar systems where the observed giant planet is closer than Jupiter to the terrestrial planet zone and may also have a large eccentricity, the process of planetary formation could be easily inhibited. The planetesimals would be ground down to dust size by mutual high velocity collisions and the remnant dusty disk would be quickly cleaned out by gaseous friction or by Poynting-Robertson drag.

A key problem in this respect is the timing of the giant planet formation. In the standard "core-accretion" model the formation of a Jupiter size planet requires about $10^{7}$ years (Pollack et al. 1996). During this timespan, the planetesimals in the terrestrial region can grow almost unperturbed, undergo runaway growth and, finally, evolve into planetary embryos before the giant planet completes its formation process and it begins to extend its gravitational reach down to the inner regions of the system. In particular, if these giant planets did form further away from the star and migrated later on to their actual position, terrestrial planets had additional time to accrete. The eccentricity stirring and the increase in the relative velocity caused by the incoming giant planet would perturb a population of Moon-size planetary embryos whose gravitational binding energy in collisions would overcome the escape energy. Each collision would lead to accumulation and the planetary formation process could be completed. In this case, the inner disc accretion process would probably reach completion without being affected by the external planet(s).

However, alternative theories on the formation of giant planets assume that they formed more rapidly by gravitational instabilities in the gaseous disk (Boss 2000) or by direct fragmentation during the protostellar collapse. Boss (2001) suggested that objects down to $1 M_{\text {jup }}$ could form in this stellar way, and thus they should be rather considered as "sub-brown dwarf stars". Such rapidly formed objects could exert strong gravitational perturbations on a planetesimal disk made of small bodies, assuming that the process of planetesimal formation itself was possible in such an environment. These perturbations might have accelerated the mutual relative velocities beyond the limit for accretion precluding the formation of larger bodies. Dissipative processes as gas drag, dynamical friction and inelastic collisions have a damping effect on the mutual encounter velocity but the increase in eccentricity due to the presence of the giant planet might have been so strong as to halt anyway the planetesimal accretion process. The migration of the planet from an exterior orbit to that presently observed may have given some additional time to the planetesimals to grow, but probably not enough to allow the formation of large embryos able to survive high velocity impacts.

In this paper we analyze the planetary formation processes in the terrestrial region for two different scenarios: 1) an early-formed giant planet is present since the early phases of planetesimal accumulation in the terrestrial region 2) the giant planet formed according to the core accretion model and lunar-size embryos could grow before the completion of the giant planet and the onset of its gravitational perturbations. We concentrated on two systems: 47 UMA and Epsilon Eridani, which, among the systems with a distant planet, are the ones having the least massive companions. The inner giant planet in 47 UMA has a semi-major axis of $2.11 \mathrm{AU}$ and an eccentricity of 0.096 (Butler \& Marcy 1996), relatively low compared to the other systems with an external planet. A second planet has been recently detected at $3.73 \mathrm{AU}$ with a mass about 3 times smaller, almost the same ratio as between Jupiter and Saturn. This system is a good candidate for investigating the planetary formation process in the terrestrial planet region since the perturbing planet is far away and 
it has a low eccentricity. Both these peculiarities tend to reduce the gravitational perturbations on planetesimals in inside orbits, possibly generating a less turbulent environment. Moreover, it has been studied from the point of view of orbital stability by Jones et al. (2001). The second system we have analyzed, Epsilon Eridani, has been chosen since in it the giant planet is even farther away, its semimajor axis being $3.3 \mathrm{AU}$, and it has a low minimum mass, only 0.86 Jovian masses (Hatzes et al. 2000). On the other hand, it is on a highly eccentric orbit $(e=0.608)$ that could reinforce the resonant perturbations on the planetesimal disk. We should stress that there are still uncertainties about the reality of the planet around Eps. Eridani due to the fact that the Doppler behaviour of such a magnetically active star is still poorly known. Nevertheless, even if the Eps. Eridani planet does not exist, our study might be taken as applying to a typical member of the category of extrasolar systems characterized by a distant low mass companion on a very eccentric orbit.

Two different sets of simulations have been performed for each of the two extrasolar systems. We first integrate the orbits of a swarm of small planetesimals in a close circumstellar disk and compute their mutual encounter velocity distribution to explore the conditions under which an early-formed external giant perturber can possibly inhibit planet growth in the terrestrial zone. In these simulations the role of gas drag is also explored. With the second set of simulations we investigate the evolution of a population of lunar-sized embryos in the same zone under the assumption that the giant planet reached its final mass when runaway growth had already occurred in the terrestrial region.

\section{The numerical models}

\subsection{Relative velocities in the planetesimal swarm}

The numerical model used to simulate the dynamical state of the planetesimal swarm in the early stages of the extrasolar system formation is derived from the code initially developed by Thébault \& Brahic (1999) to study collisional processes in the early inner solar system. This code follows in a deterministic way the evolution of a three dimensional swarm of test particles perturbed by a massive planet. Basically, the code solves Kepler's equation for the central potential and it includes the perturbing potential by integrating Gauss' equations. It has also been improved in order to handle close encounters up to a few physical radii from the perturbing body. To speed up the computations, we will not consider the effects of collisions on the orbits of the planetesimals, while gas drag will be taken into account. We neglect collisions also because the extremely high encounter velocities we find in most of our simulations yield a high rate of catastrophic impacts with fragmentation of the colliding bodies. This would limit the propagation of excited bodies through subsequent collisions in the planetesimal disk and, consequently, reduce their stirring effects on the relative velocity distribution.
Collisions should thus mainly affect the planetesimal number density evolution rather than the encounter dynamics. Nevertheless, a more refined study should definitely address this role of collisions on dynamics that, however, is at present out of the computer ability for fully deterministic models. For sake of simplicity and to avoid the multiplication of free parameters, collisions will not be included in the present first step study and we will focus on the coupling of external gravitational perturbations and gas drag, thus following the same assumption as Marzari et al. (1997) or Kortenkamp \& Wetherill (2000). The collision search algorithm is nevertheless activated in order to compute accurate relative velocities between the bodies when a collision is expected to occur, and to obtain the statistical distribution of the impact speeds over selected intervals of time. It is worth noting that it is not possible to directly derive the encounter velocities values analytically from the local eccentricity and inclination distributions. This can be done only when the planetesimal system satisfies two restrictive conditions. 1) The angular orbital parameters are randomly distributed. This is not the case in our simulations because the secular perturbations by the planet force phased oscillations in eccentricity and strong clustering of the perihelion longitude. The relative velocity distribution may become totally decorrelated from the eccentricity distribution (Thébault \& Brahic 1999; Marzari \& Scholl 2000). 2) The encounters occur mainly between neighboring particles. Even this condition is violated in our system where the bodies, due to the large eccentricity values, have large radial excursions.

From the values of relative velocity we can estimate the minimum size of objects that can accrete in the system through the relation:

$R_{\mathrm{acc}}=f\left(\frac{3}{8 \pi \rho_{\mathrm{s}} G}\right)^{0.5} \Delta v$

where $\rho_{\mathrm{s}}$ is the number density of planetesimals in the disk and $f$ is a coefficient accounting for the energy dissipation after an impact. For the violent impacts considered here, we will adopt the usual assumption that $90 \%$ of the energy is dissipated in the radial direction (e.g. Petit \& Farinella 1993) which leads to $\langle f\rangle \simeq 0.7$. We stress here the fact that our simulations are not intended to model the accumulation process, but rather to study the dynamical conditions under which accretion could be favored or precluded.

Gas drag is taken into account as an additional force $F_{\mathrm{g}}$ in Gauss' perturbing equations. The expression for $F_{\mathrm{g}}$ is valid for large Reynolds number and is derived from Weidenschilling \& Davis (1985) and Marzari et al. (1997):

$\boldsymbol{F}_{\mathrm{g}}=-\frac{3 \rho_{\mathrm{gas}} C_{\mathrm{d}}}{8 \rho_{\mathrm{s}} R_{\mathrm{r}}} \quad v_{\mathrm{rel}} \boldsymbol{v}_{\mathrm{rel}}$

where $\rho_{\text {gas }}$ is the gas density, $C_{\mathrm{d}}$ a coefficient which depends on particles geometry, $R_{\mathrm{r}}$ the physical radius of planetesimals and $v_{\text {rel }}$ the relative velocity between gas and planetesimals. As a first approximation, we adopt here 
a gas density profile proposed by Hayashi (1981) for the early solar nebula, with $\rho_{\text {gas }}=1.35 \times 10^{-9} r^{-2.75} \mathrm{~g} \mathrm{~cm}^{-3}$. We also take $C_{\mathrm{d}}=0.4$, the approximate value for spherical bodies and $\rho_{\mathrm{s}}=3 \mathrm{~g} \mathrm{~cm}^{-3}$. Following Marzari et al. (1997) we also take into account the modified keplerian velocities of gas streamlines due to the pressure gradient. This difference in Keplerian velocities is given by $\Delta v_{\mathrm{k}}=5 \times 10^{-3} v_{\mathrm{k}}$. The nominal gas drag simulations consider planetesimals of size $R_{\mathrm{r}}=7 \mathrm{~km}$. However, runs for bigger objects with $R_{\mathrm{r}}=70 \mathrm{~km}$ have also been performed.

In each simulation for the two extrasolar planet systems 47 UMA and Epsilon Eridani we numerically integrated the trajectories of 2000 test particles in three dimensions orbiting around the star with initial eccentricities and inclinations chosen in order to have initial encounter velocities of the order of $20 \mathrm{~m} \mathrm{~s}^{-1}$. This is the average value expected in a swarm of 5 to $10 \mathrm{~km}$ planetesimals at equilibrium. The other orbital parameters were randomly selected. The simulations were carried out over timescales ranging from $10^{5}$ to $10^{6}$ years, typical for the planetesimal accretion process.

\subsection{Large embryos accumulation}

To model the final stage of terrestrial planet formation in the hypothesis that planetary embryos had enough time to form before the completion of the giant planet or before its migration into an inner orbit, we adopted the hybrid symplectic algorithm by Chambers (1999). A timestep of 0.5 days was used to account for the fast evolution of planetesimals in orbits so close as 0.4 AU from the star. We integrated the orbits of 210 planetary embryos with semimajor axes ranging from 0.4 to $1.4 \mathrm{AU}$, eccentricities all initially set to 0 and inclinations ranging from 0 . to $0.5^{\circ}$. The mass of the embryos was $m=7.3 \times 10^{22} \mathrm{~kg}$ (Moon mass) and the bulk density $3 \mathrm{~g} \mathrm{~cm}^{-3}$. An initial separation of two mutual Hill's spheres is adopted for the planetary embryos and their total mass is about $75 \%$ of the mass expected for an initial planetesimal swarm that extends from 0.4 to $1.4 \mathrm{AU}$. We assume a surface density that varies as $a^{-3 / 2}$ and that at $1 \mathrm{AU}$ has a value equal to $16.7 \mathrm{~g} \mathrm{~cm}^{-2}$ in agreement with Wetherill \& Stewart (1993). This assumption is arbitrary, since we have no idea of the mass and size of the accretion disks that surrounded the two stars 47 UMA and Epsilon Eridani. However, as we will see, the conclusions about the occurrence of planetary formation are robust and almost independent of the initial conditions.

\section{Results}

\subsection{Epsilon Eridani}

The Epsilon Eridani simulations consider 1000 particles initially spread between 0.1 and $1.2 \mathrm{AU}$ from the star. The Epsilon Eridani system turns out to be an unfavorable environment for planetesimal accretion in the inner zones of the circumstellar disk if the external planet

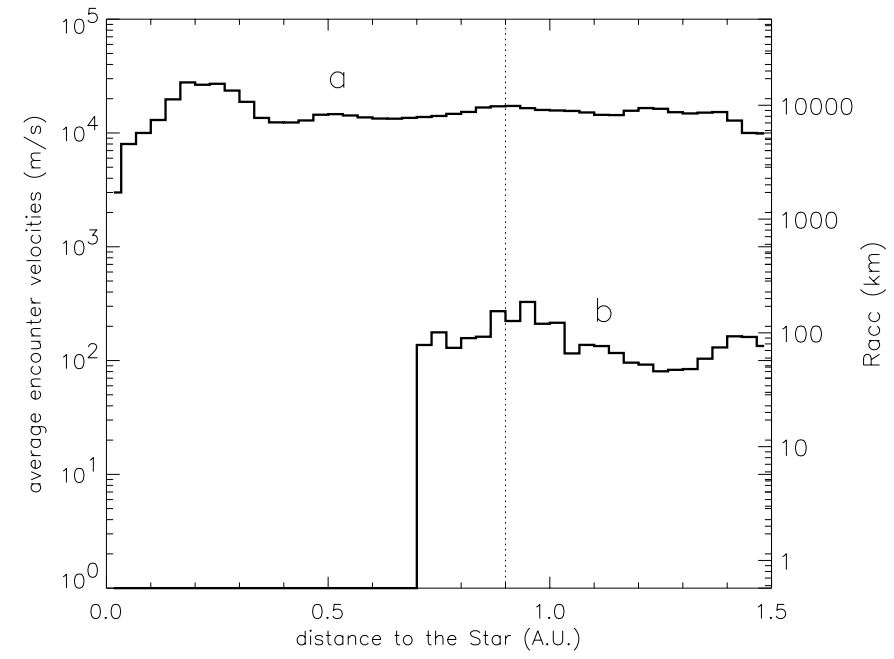

Fig. 1. Encounter velocity distribution for planetesimals in the Epsilon Eridani system at $t=10^{5}$ years. Line a) refers to the simulation without gas drag, line b) to the simulation with gas drag on $7 \mathrm{~km}$ planetesimals. The dotted vertical line marks the outer limit of the stable region. The encounters in the region beyond $0.9 \mathrm{AU}$ are due to objects with semi-major axis $a<$ $0.9 \mathrm{AU}$ but with large eccentricities so that their aphelia are outside $0.9 \mathrm{AU}$. In case b, the velocity between 0 . and $0.7 \mathrm{AU}$ appears to be zero because the region has been rapidly cleared out by gas drag. $R_{\text {acc }}$ is given by Eq. (1).

formed before the onset of runaway growth in the terrestrial region. This is true notwithstanding the low mass and large distance of the external giant planet. The numerical simulations show clearly that the planet's highly eccentric orbit forces extremely large oscillations in the eccentricity of the planetesimals even for the lowest possible mass of the planet, i.e. $0.86 M_{\text {jup }}$. Due to the large induced eccentricities a significant fraction of the planetesimal swarm becomes rapidly unstable because of repeated close encounters with the giant planet. The whole region beyond $0.9 \mathrm{AU}$ is cleared so rapidly that it does not have the time to affect the relative-velocity dispersion in the inner region of the disk. An additional simulation with the planetesimals initially distributed only in the region within $a<0.9$ AU where the individual orbits are stable gave similar results, confirming that the scattered planetesimals do not significantly alter the accretion process in the relatively "calm" inside region.

Even in the inner stable region of the planetesimal disk, inside $0.9 \mathrm{AU}$, the dynamical conditions for accretion are nevertheless very extreme and planetary formation not likely. Large relative velocities build up everywhere between planetesimals exceeding by far $3000 \mathrm{~m} \mathrm{~s}^{-1}$, i.e. the accretion limit for $1700 \mathrm{~km}$ objects (Fig. 1). The gas drag tends to damp the relative velocities towards lower values, of the order of $100 \mathrm{~m} \mathrm{~s}^{-1}$, thanks also to the strong alignment of the perihelia (Marzari \& Scholl 2000). However, the coupling of the high gas density with the large orbital eccentricities forced by the giant planet induces a fast spiraling of the bodies towards the star. Only a small fraction of the planetesimals survive at the end of the simulation 
after $10^{5} \mathrm{yr}$ while more than $95 \%$ of them end up into the star. The relative velocities shown in Fig. 1 concern the few bodies still orbiting the star beyond $0.7 \mathrm{AU}$ at the end of the simulation.

If the planet completed its growth at later times and planetary embryos had enough time to form in the inner part of the Epsilon Eridani system, they can accumulate into larger bodies within $0.8 \mathrm{AU}$. Beyond this value the bodies, excited by the gravitational perturbations of the external planet, mostly end up impacting on the star while a minor fraction is ejected from the system. The limit of $0.8 \mathrm{AU}$ for accumulation is closer to the star compared to the value of $0.9 \mathrm{AU}$ observed in the simulation for smaller planetesimals. This might be due to the different timescales and impact frequencies involved in the simulations. In the inner regions accumulation occurs on a short timescale of the order of a few million of years. In Fig. 2 we show the embryos evolution at three different times $t=5 \times 10^{5}, 1.5 \times 10^{6}$, and $2.7 \times 10^{6}$ years, respectively. We enlarge in the figure the region between 0.3 and $0.8 \mathrm{AU}$ where accumulation occurs; all bodies orbiting beyond $0.8 \mathrm{AU}$ are ejected or impact on the sun. The embryos populating at the beginning this region accrete in two final large bodies. The one inside has a mass about $76 \%$ of the Earth's mass, a semi-major axis of $0.32 \mathrm{AU}$ and a low eccentricity, 0.17 . The second planet is farther out at $\mathrm{a}=0.51 \mathrm{AU}$ and its mass is lower, around $28 \%$ of the Earth's mass, while its eccentricity is similar to that of the inner planet, about 0.19 .

The rapid accumulation process is favored by the large eccentricities excited by the external planet. While a large average eccentricity can halt the accumulation of small planetesimals, it can instead increase the impact probability among embryos and speed up their accretion in a planetary body. The disruption threshold for embryoembryo collisions is in fact much higher, due to their high gravitational binding energy, compared to that of planetesimals.

As for the small planetesimal case, we performed an additional numerical simulation where we start the planetary embryos within $0.8 \mathrm{AU}$, the region of stable orbits. The total mass in the disk is scaled down and we have the same initial orbital separation between the embryos in the two simulations. The slow growth of the giant planet could have caused a progressive clearing of bodies beyond $0.8 \mathrm{AU}$ either by a slow onset of instability or by high velocity collisions. If this was the case, large embryos could not have formed at distances larger than $0.8 \mathrm{AU}$. In this second simulations (Fig. 3) the two final planets at the end of the accumulation phase are slightly more massive: $92 \%$ of the Earth mass the inner one with semimajor axis $a=0.32 \mathrm{AU}$, and $50 \%$ for the outer one with $a=0.49 \mathrm{AU}$. The average eccentricities, computed over a timescale of $1 \mathrm{Myr}$, are slightly smaller than the previous case. The largest fraction of mass ending up in planets is higher in this case. In the previous simulation those embryos orbiting beyond $0.8 \mathrm{AU}$ and scattered into high eccentric orbits by the perturbation of the giant planets, had enough time
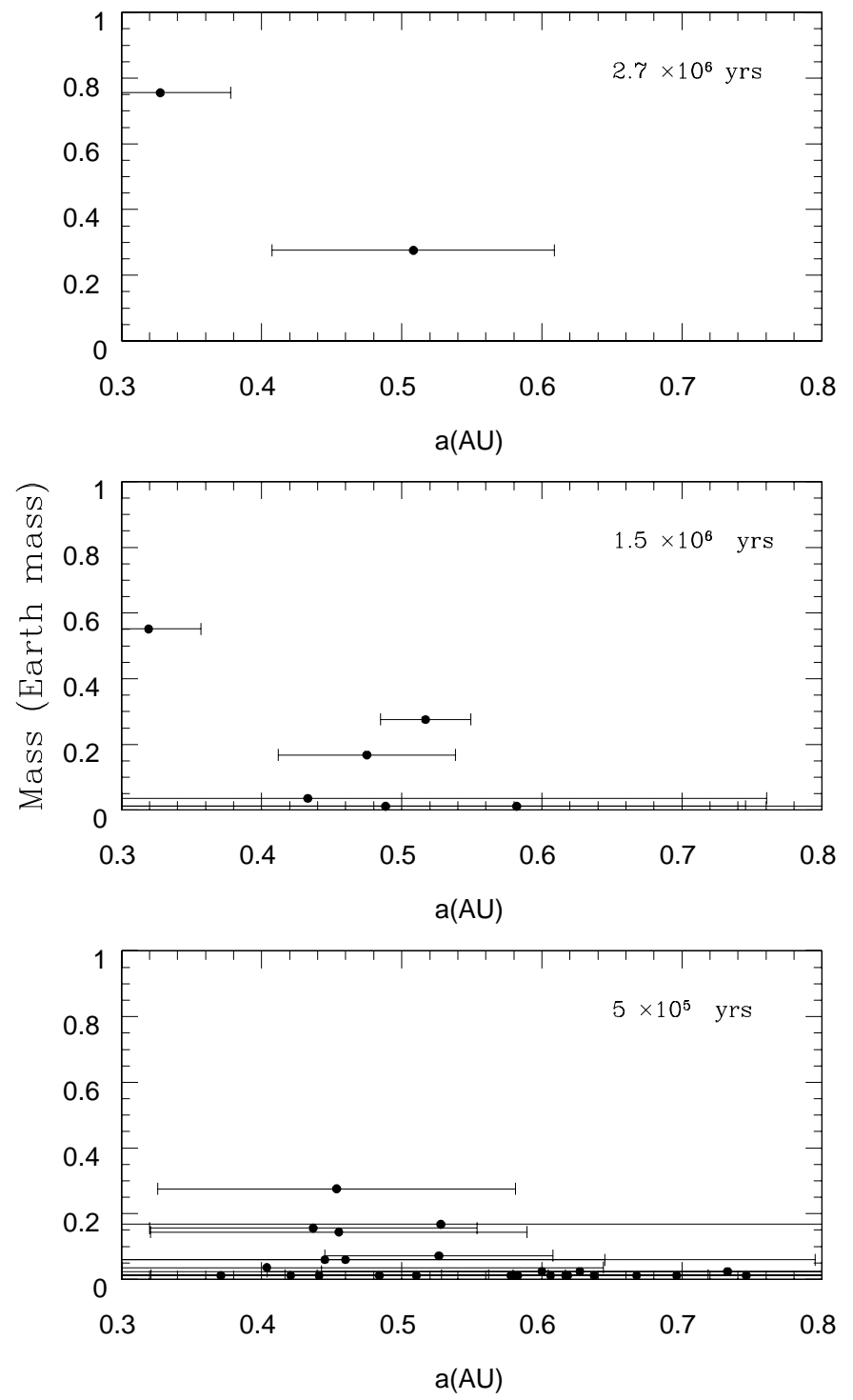

Fig. 2. Evolution of the embryos' mass and orbit in the simulated Epsilon Eridani system at three different times: $5 \times 10^{5}$, $1.5 \times 10^{6}$, and $2.7 \times 10^{6} \mathrm{yr}$. For each data point the distance from perihelion to aphelion is represented by the error bar.

to stir the relative velocities between the bodies in the inner region and cause a largest fraction of them to impact either the star or the giant planet.

\section{2. $47 U M A$}

In the first simulation for 47 UMA we considered only the inner giant planet as perturber, neglecting the outer planet. This allows us to evaluate separately the influence of the second planet and also to give general results for systems with a single massive planet on a low-eccentricity orbit far from the star. The first 47 UMA simulation considers 2000 test particles initially spread between 0.1 and 5.5 AU from the central star. The 47 UMA giant planet is more massive compared to that of Epsilon Eridani but it has a moderately low eccentricity. Jones et al. (2001) identified an unstable area extending down to $1.3 \mathrm{AU}$ for 

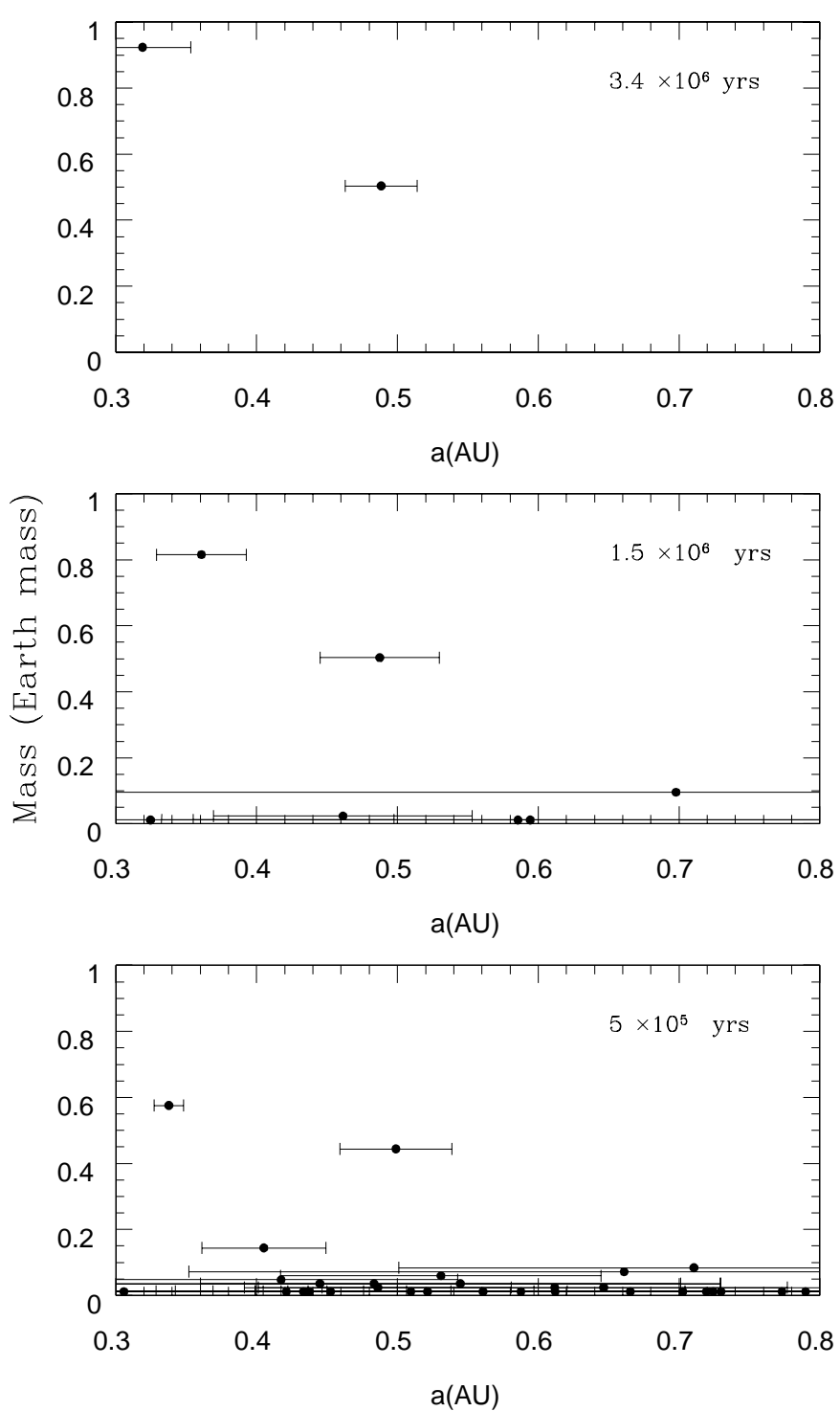

Fig. 3. The same as in Fig. 2 but with an initial population of embryos distributed within $a=0.8$ AU. Notice the lower eccentricities and higher masses of the final planets.

the minimum mass $\left(2.4 M_{\text {jup }}\right)$ case corresponding to the orbit of the planet viewed edge-on. This unstable region is also clearly visible in our low-mass gas-free run (Fig. 4), though it extends as far as 1.45 AU. This discrepancy on the stability outer edge is related to the shorter time scales spanned in our simulations. We integrate the planetesimal orbits for a few $10^{5}$ years while the Jones et al. simulation covers an interval of time of $\left(10^{9}\right.$ years $)$. This is an important point in particular when we consider the effects of the 2:1 resonance at $1.33 \mathrm{AU}$ which proved to be unstable over a time scale of $10^{8}$ years. Another difference between our results and those of Jones et al. is probably related to our more restrictive condition for exclusion after a close encounter with the planet, i.e. $1 / 3 R_{\mathrm{H}}$ (Hill Radius) instead of $3 R_{\mathrm{H}}$ adopted by Jones et al. It is interesting to note the narrow stable region corresponding to the $3: 2$ resonance at $1.61 \mathrm{AU}$ where three objects survive. This is an illustration of the classical resonant protection mechanism, similar to that observed for the 2:3 resonance

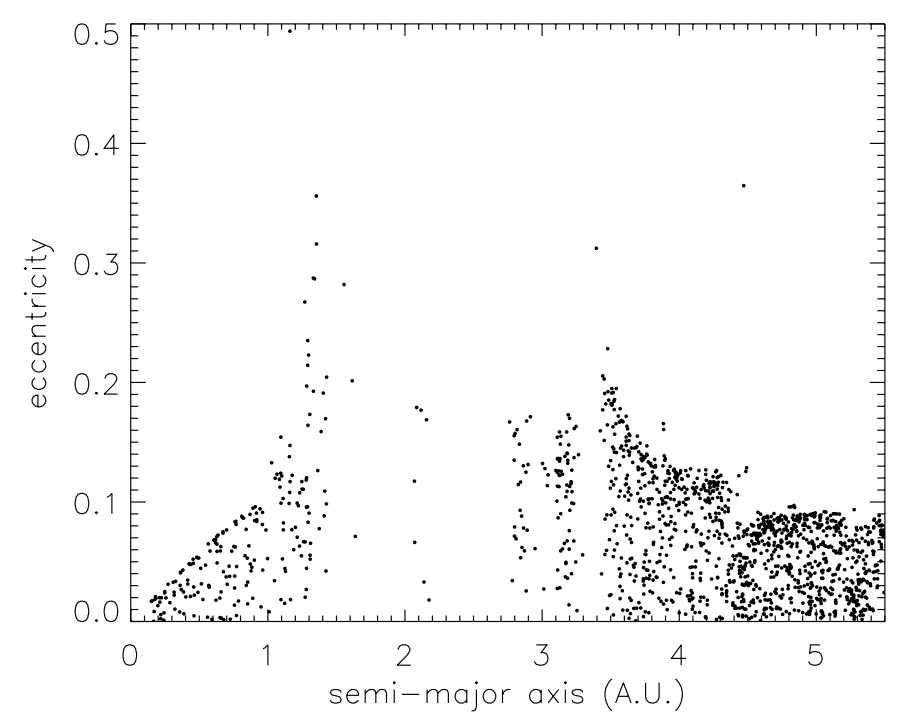

Fig. 4. (e, a) diagram at $t=10^{5}$ years for the $47 \mathrm{UMa}$ system with a $2.4 M_{\text {jup }}$ perturbing planet and no gas drag.

in the Kuiper belt. Note also the group of stable objects at 2.1 AU, corresponding to bodies in the 1:1 corotation resonance with the planet, that may resemble the Trojan population in our solar system.

High encounter velocities between the planetesimals were established very quickly in the inner disk, already occuring after $10^{4}$ years (Fig. 5) and it does not show significant changes for a few $10^{5}$ years. The high relative velocity regime is mostly due to encounters with bodies originating from the highly excited 0.9 to $1.4 \mathrm{AU}$ region, and, in particular, from the strong 2:1 resonance. The wide radial excursion of these bodies on highly eccentric orbits drives them to the innermost regions of the disk. This generates an environment which is relatively hostile to the accretion process of small to medium size planetesimals. The minimum size of the bodies which could accrete in spite of the high velocity impacts varies from 1500 to $500 \mathrm{~km}$ between 1.4 and $0.3 \mathrm{AU}$. There is a sharp transition towards small relative velocities below 0.3 AU principally because this region is safe from encounters with the high eccentricity bodies originating from the perturbed 0.9-1.4 AU region. In this innermost area objects as small as $10 \mathrm{~km}$ can have accreting encounters. In the outer disk relative velocities have values ranging from $2000 \mathrm{~m} \mathrm{~s}^{-1}$ at $2.8 \mathrm{AU}$ to $500 \mathrm{~m} \mathrm{~s}^{-1}$ at $5.5 \mathrm{AU}$, the outer edge of the system we considered in our simulation. These velocities do not prevent the accretion of bodies bigger than 280 to $1100 \mathrm{~km}$, but they inhibit the growth of smaller objects.

We also explored a case with a more massive perturber, a $4.8 M_{\text {jup }}$ planet corresponding to the possible case in which we are observing the orbit of the planet in a plane tilted by 60 degrees towards the line of sight. The unstable region covers approximately the same radial range compared to the low mass case with only small differences related to the slight shift of the resonances caused by the larger mass of the planet. The relative velocities increase on average by $20 \%$ and, as a consequence, the constrains on the planetesimal accumulation process are practically the same as in the $2.4 M_{\text {jup }}$ case. 


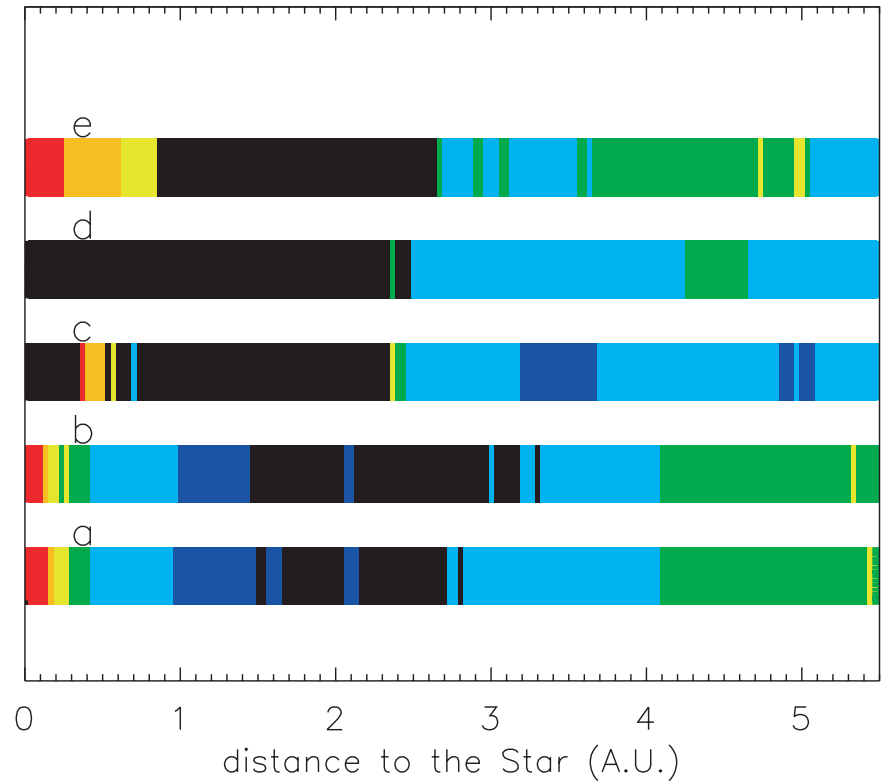

Fig. 5. Average encounter velocity distribution in the $47 \mathrm{UMa}$ system for 5 different cases.

a) At $t=10^{5}$ years with $M_{\text {plan }}=2.4 M_{\text {jup }}$ and no gas.

b) Same as a) with $M_{\text {plan }}=4.8 M_{\text {jup }}$.

c) Same as a) with gas drag on $7 \mathrm{~km}$ objects at $t=5 \times$ $10^{4}$ years.

d) Same as c) at $t=10^{5}$ years.

e) Same as a) with gas drag on $70 \mathrm{~km}$ objects at $t=10^{5}$ years. The color scaling reads as follows:

red: $\left\langle d v><25 \mathrm{~m} \mathrm{~s}^{-1}\right.$, i.e. $R_{\text {acc }}=15 \mathrm{~km}$;

orange: $\left\langle d v><100 \mathrm{~m} \mathrm{~s}^{-1}\right.$, i.e. $R_{\text {acc }}=60 \mathrm{~km}$;

yellow: $<d v><500 \mathrm{~m} \mathrm{~s}^{-1}$, i.e. $R_{\text {acc }}=300 \mathrm{~km}$;

green: $\left\langle d v><1300 \mathrm{~m} \mathrm{~s}^{-1}\right.$, i.e. $R_{\mathrm{acc}}=750 \mathrm{~km}$;

pale blue: $\left\langle d v><2500 \mathrm{~m} \mathrm{~s}^{-1}\right.$, i.e. $R_{\mathrm{acc}}=1400 \mathrm{~km}$;

dark blue: $\left\langle d v>>2500 \mathrm{~m} \mathrm{~s}^{-1}\right.$;

black: empty region.

(See text for details.)

When we introduce gas drag in the numerical simulations to model the behaviour of small planetesimals, the dynamical evolution of the system is drastically different, particularly in the inner region of the disk where the gas density is higher. The eccentricities are rapidly damped and the planetesimals quickly spiral towards the star. The inner regions within 0.5 AU are progressively cleared out after $5 \times 10^{4}$ years and the inner disk is totally void after $10^{5}$ years (Fig. 6). However, before this final stage is reached a small fraction of the inner portion of the disk is populated by planetesimals with very low eccentricities and low encounter velocities, between 20 and $100 \mathrm{~m} \mathrm{~s}^{-1}$ (Fig. 5). In this region and for a limited timespan, accretion of kilometer-sized planetesimals is possible. Even the outer disk is significantly affected by gas drag but in a different way. Figure 6 shows the strongly nonhomogeneous structure of the outer region after $10^{5}$ years where strong overdensities appear at the 3:2, 5:3, and 5:2 resonance locations at 2.8, 2.96 and $3.9 \mathrm{AU}$, respectively. The resonant capture in presence of a dissipative force determines a concentration of bodies at the resonances as described in Scholl et al. (1993) and Weidenschilling \& Davis (1985). Apart from the resonance locations, the outer disk

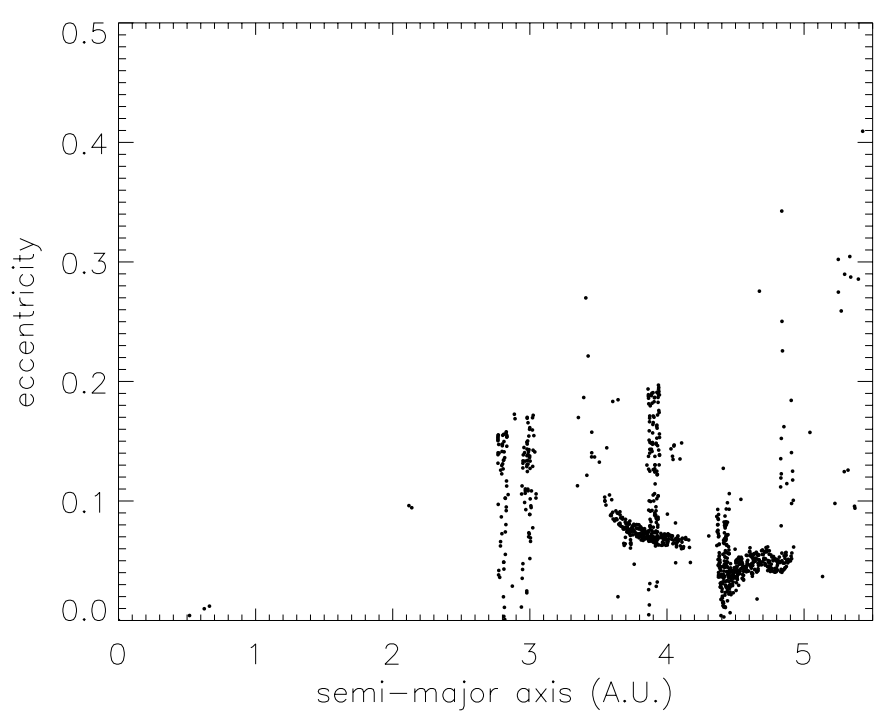

Fig. 6. Same as Fig. 4 with gas drag and $7 \mathrm{~km}$ planetesimals.

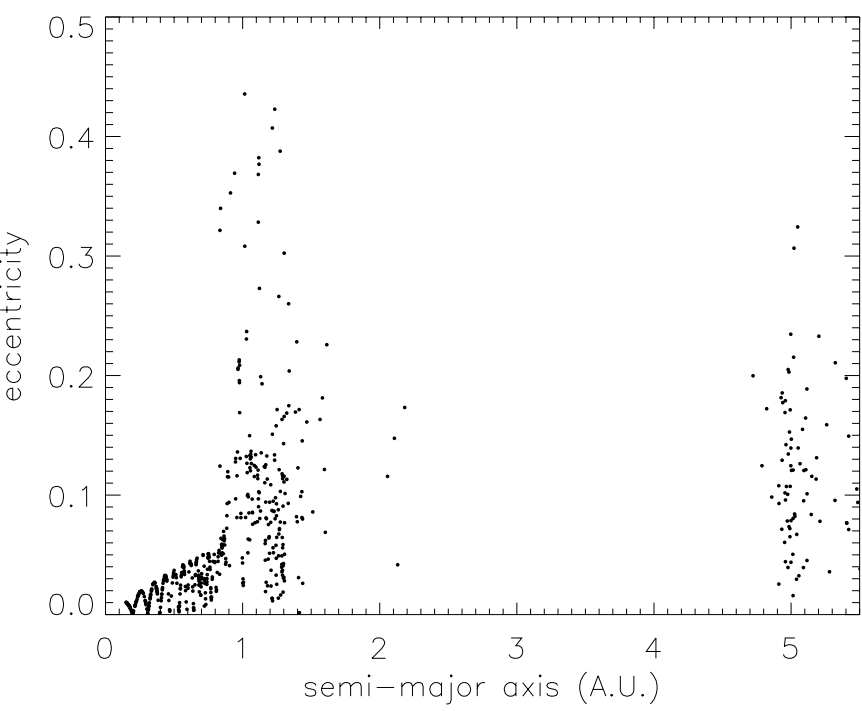

Fig. 7. Same as Fig. 4 but with the presence of a second planet of mass $0.76 M_{\text {jup }}$ with initial orbital parameters $a=3.73 \mathrm{AU}$ and $e=0.1$. Mutual perturbations between the 2 planets are taken into account and the 2 bodies are assumed to be coplanar.

is almost totally depleated of test bodies. Among the surviving bodies not in resonance there is a strong periastron alignment (resulting in a very narrow eccentricity dispersion) due to the coupling between gas drag and forced oscillations induced by an eccentric perturber (Marzari \& Scholl 2000).

Larger planetesimals (we considered $70 \mathrm{~km}$ size test bodies) are less affected by frictional forces and after $10^{5}$ years the inner disk within $0.9 \mathrm{AU}$ is still populated (Fig. 5). Moreover, the periastra are all in phase and low encounter velocities are maintained even for these larger bodies. In the region $a<0.75 \mathrm{AU}$, all the relative speds are below $150 \mathrm{~m} \mathrm{~s}^{-1}$, the approximate critical value for accretion vs. disruption in a $70 \mathrm{~km}$ planetesimal population.

A second planet has been recently discovered around 47 UMA (Fischer et al. 2001) with a $M \cdot \sin (i)$ of $0.76 M_{\text {jup }}$ at 3.73 AU from the central star. Its orbital eccentricity 
is not well constrained yet, at present ranging between 0 and 0.2 . Nevertheless, preliminary simulations have been carried out in order to estimate the influence of this additional body. The direct effect of the second planet's perturbations is, as could be logically expected, to clear out a large fraction of the outer disk (Fig. 7). In the inner disk the influence of the second planet is much weaker compared to that of the first planet since it is farther away from the star and it has also a lower mass. However, it induces secular oscillations of the orbital parameters of the first more massive planet, as does Saturn with Jupiter, and it indirectly influences even the inner regions of the disk. The main difference with respect to the one-planetonly case is the presence of highly excited bodies (with eccentricities up to 0.4 ) in the $4: 1$ and $3: 1$ mean motion resonances at 0.83 and $1.00 \mathrm{AU}$ respectively. As a consequence, the encounter velocities are significantly increased in the $0.35<a<1.4 \mathrm{AU}$ region. Nevertheless, this velocity increase does not strongly change the results presented in the previous chapter, since the regions beyond $0.35 \mathrm{AU}$ were anyway already strongly excited in the one-planet case (cf. Fig. 5).

The outcome of the $N$-body integration of large planetary embryos with the single giant inner planet in the 47 UMA system is shown in Fig. 8. As in the Epsilon Eridani system, most of the bodies orbiting beyond $1 \mathrm{AU}$ are rapidly ejected out of the system or impact the star within the first 1 Myr. Even in this case terrestrial planets can grow only very close to the star. After about $1.1 \times 10^{7}$ years two planets form, one at 0.47 with a mass $62 \%$ of the Earth mass, and one at $0.7 \mathrm{AU}$ with a mass $20 \%$ of the Earth mass, on a timescale longer compared to the Epsilon Eridani case due to the lower eccentricities induced by the external perturbing planet. The average final eccentricities of the two planets are low, 0.06 for the inner one, 0.08 for the outer, the inclinations are $2.25^{\circ}$ and $3.57^{\circ}$, respectively. If we consider a reduced initial population of embryos from which we excluded all those bodies with orbits becoming unstable after a short timespan, the results do not change significantly. Even when we add the second planet in the simulation, again we retrieve a terrestrial planet system that is statistically similar to that observed in the first simulation (see Fig. 9). The only difference is the formation of three planets instead of two, but the orbital parameters are similar and the final mass in the terrestrial planets is comparable to that of the first simulation. The stability of this three-planet system has been tested for additional $15 \mathrm{Myr}$ and no encounter between them occurred during this time. The three planets are close to high order mutual commensurabilities and this might increase the stability of the system. The inner planet is located at $0.42 \mathrm{AU}$ and it has a mass of $0.36 M_{\oplus}$, the middle one is at $0.60 \mathrm{AU}$ with a mass of $0.40 M_{\oplus}$, and the outer one is at $0.75 \mathrm{AU}$ with a mass of $0.11 M_{\oplus}$.

\section{Discussion and conclusions}

From our simulations, it appears that an early-formed giant planet should have prevented any planetesimal
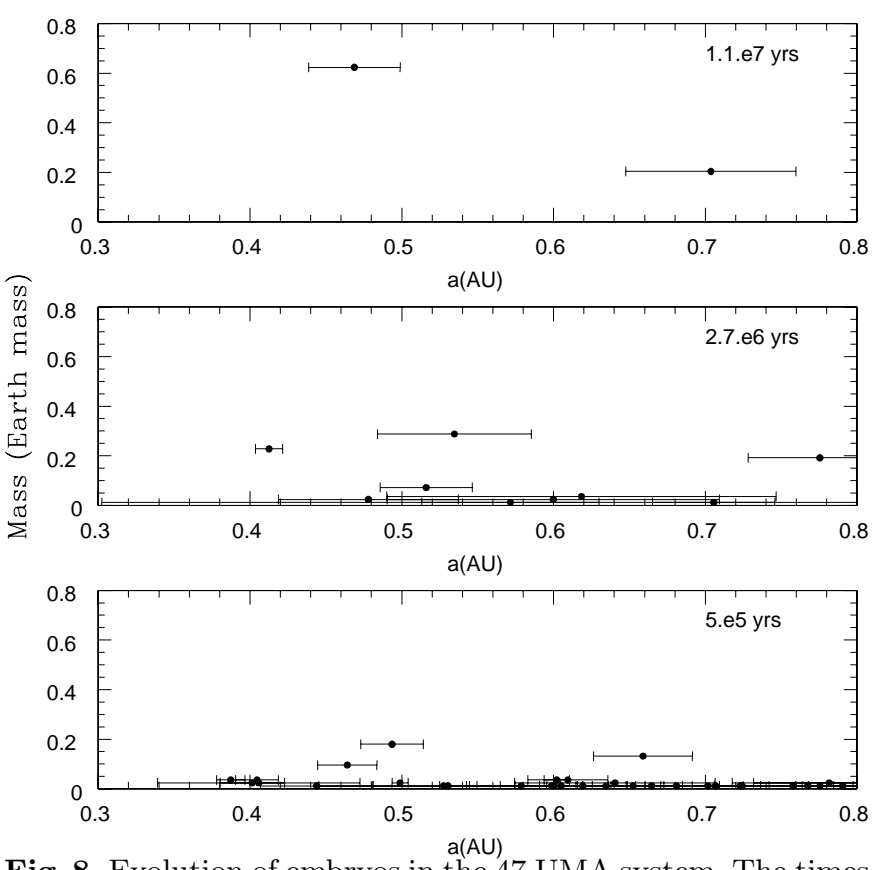

Fig. 8. Evolution of embryos in the 47 UMA system. The times are $5 \times 10^{5}, 2.7 \times 10^{6}$, and $1.1 \times 10^{7} \mathrm{yr}$.
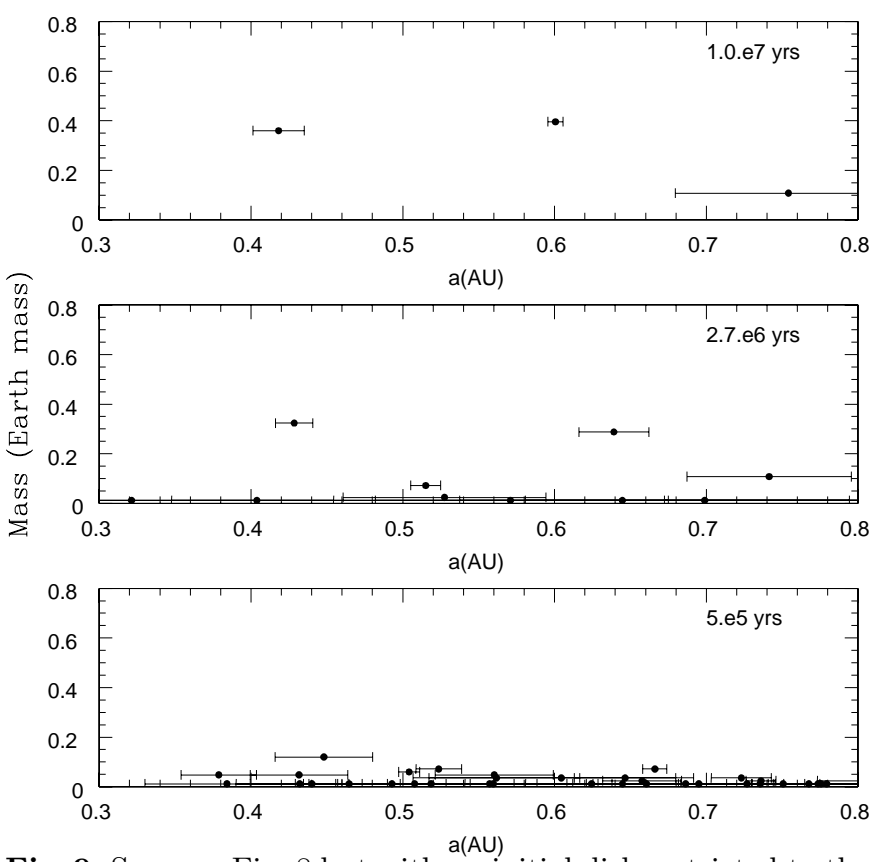

Fig. 9. Same as Fig. 8 but with an initial disk restricted to the stable region below $1 \mathrm{AU}$.

accretion in the inner regions of the Epsilon Eridani system. It is hardly possible that large planetary embryos could have developed because of a very hostile environment where either large impact velocities are excited by the planet gravitational perturbations and collisions are mostly disruptive, or the gas friction disperses and clears the inner regions even in the case where the lowest mass is assumed for the planet. In the alternative scenario where the giant planet formed slowly giving a chance to the planetary embryos to form in a quiet environment, then the final stage of planetary accretion, where the large embryos collide to form the terrestrial planets, could have occurred even in presence of the fully formed giant planet. In this 
case the final system would resemble our solar system with a giant planet in an external orbit and smaller rocky planets in the inner regions. It is interesting to note as the large eccentricities of the embryos excited by the giant planet would have accelerated the final stage of planet formation reducing the timescale of $10^{8}$ years estimated for the Solar System (Chambers \& Wetherill 1998) to only a few million of years.

For the 47 UMa system we found that in absence of gaseous friction a fully formed giant planet should inhibit accretion of an initial swarm of planetesimals almost everywhere in the disk, apart from a very small inner region. It is worth noticing that this result is relatively robust and it depends only weakly on the size of the initial planetesimals. The gradient in the relative velocities between the planetesimals from the inner quiet region to the strongly perturbed outer zone is very sharp and the outer limit for accretion of 10 kilometer bodies, $0.2 \mathrm{AU}$, is very close to the accretion limit for 500 kilometer objects, $0.4 \mathrm{AU}$ (Fig. 5). This size range covers any "reasonable" value for an initial population of planetesimals in the terrestrial zone. Note that the inhibition of planetesimal accretion holds also for a large fraction of the outer disk, at least until 5.5 AU, the outer limit for our simulations. The influence of the second and more distant planet that might have been recently discovered does not significantly affect these results. It adds excitation to regions which were already strongly hostile to planetesimal accretion in the one-planet case and does not affect the small quiet innermost region of the disk.

When the gas drag force is included in our simulations, there is a short timespan (a few $10^{4}$ years) where accretion of kilometer-sized objects could be possible in a narrow region of the inner disk, i.e. between 0.3 and $0.6 \mathrm{AU}$. If bodies larger than $70 \mathrm{~km}$ had enough time to form in this band, then they might survive to the subsequent phase of higher velocity impacts and maybe form planetary embryos by runaway growth. In fact our simulations show that for these $70 \mathrm{~km}$ bodies the accretion process tends to be enhanced by the damping effect of gaseous friction (Fig. 5). Nevertheless, these results are only indicative since they strongly depend on several poorly constrained parameters such as the gas density in the early disk. We assumed in our simulation a density typical for the solar system, but this assumption is highly hypothetical and other density values and profiles may be equally plausible. If for example the gas density was an order of magnitude lower, then the evolution of the $7 \mathrm{~km}$ population would have been comparable to that found for the $70 \mathrm{~km}$ population in our nominal gas drag run. As a consequence, accretion of kilometer-sized objects would be possible in the whole $a<0.5$ AU region for more than $10^{5}$ years (Fig. 5). On the other hand, higher gas densities would prevent accretion by rapidly clearing the inner zone. Another crucial parameter is the initial size of the planetesimals. If bodies as big as 50-100 km are present in the initial gaseous disk, then our simulations show that they can directly accrete into large bodies. An initial range between 1 to $10 \mathrm{~km}$ seems reasonable in the inner region of a protoplanetary disk, but larger values cannot be completely ruled out due to the uncertainties on the planetesimal forming mechanisms (e.g. Wurm et al. 2001). It is also a critical issue whether planetesimals could actually form in presence of an early formed giant planet.

Very different is the scenario of terrestrial planet formation when the giant planet reached its final mass only after the runaway growth of embryos in the inner regions. This would be the case of a giant planet forming via the standard core-accretion model. In the Epsilon Eridani system, the embryo accumulation process would lead to the formation of one or two planets inside $0.8 \mathrm{AU}$ with masses comparable to those of Venus and Mars but on a shorter timescale compared to that expected for the Solar System planets. A similar results is obtained for the 47 UMA system even if the terrestrial planetary formation process is complicated by the presence of a second giant planet. In spite of the secular perturbations induced by a system with two giant planets, small Venus-size planets can form on a timescale of the order of $10 \mathrm{Myr}$.

Acknowledgements. The authors would like to thank J. Lissauer for fruitful refereeing and comments.

\section{References}

Boss, A. P. 2000, ApJ, 536, L101

Boss, A. P. 2001, ApJ, 551, L167

Butler, R. P., \& Marcy, G. W. 1996, ApJ, 464, L153

Chambers, J. E. 1999, MNRAS, 304, 793

Chambers, J. E., \& Wetherill, G. W. 1998, Icarus, 136, 304

Charnoz, S., Thébault, P., \& Brahic, A. 2001, A\&A, 373, 683

Fischer, D., Marcy, G., Butler, P., Laughlin, G., \& Vogt, S. 2001, ApJ, submitted

Hayashi, C. 1981, PthPS, 70, 35

Hatzes, A. P., Cochran, W. D., McArthur, B., et al. 2000, ApJ, 544, L145

Jones, B. W., Sleep, P. N., \& Chambers, J. E. 2001, A\&A, 366, 254

Kortenkamp, S. J., \& Wetherill, G. W. 2000, Icarus, 143, 60

Lin, D. N. C., Bodenheimer, P., \& Richardson, D. C. 1996, Nature, 380, 606

Lissauer, J. J. 1993, ARA\&A, 31, 129

Marzari, F., Scholl, H., Tomasella, L., \& Vanzani, V. 1997, Planet. Space Sci., 45, 337

Marzari, F., \& Scholl, H. 2000, ApJ, 543, 328

Murray, N., Hansen, B., Holman, M., \& Tremaine, S. 1998, Science, 279, 69

Petit, J.-M., \& Farinella, P. 1993, CeMDA, 57, 1

Pollack, J. B., Hubickyj, O., Bodenheimer, P., et al. 1996, Icarus, 124, 62

Scholl, H., Roques, F., \& Sicardy, B. 1993, CeMDA, 56, 381

Thébault, P., \& Brahic, A. 1999, Planet. Space Sci., 47, 233

Ward, W. R., \& Hourigan, K. 1989, ApJ, 347, 490

Weidenschilling, S. J., \& Davis, D. R. 1985, Icarus, 62, 16

Weidenschilling, S. J., \& Marzari, F. 1996, BAAS, 28, 12.14

Wetherill, G. W., \& Stewart, G. R. 1993, Icarus, 106, 190

Wurm, G., Blum, J., \& Colwell, J. E. 2001, Icarus, 151, 318 